\title{
Art and Technology for Young Creators
}

\author{
Marikken Høiseth $^{1}$ and Letizia Jaccheri ${ }^{2}$ \\ ${ }^{1}$ Department of Product Design, \\ Norwegian University of Science and Technology (NTNU), \\ Trondheim 7491, Norway \\ marikken.hoiseth@ntnu.no \\ ${ }^{2}$ Department of Computer and Information Science, NTNU, \\ Trondheim 7491, Norway \\ letizia@idi.ntnu.no
}

\begin{abstract}
The general research goal that motivates this work is the aim to produce new knowledge at the intersection between art and technology. The practical goal of the project reported in this paper is to strengthen pupils' interest in computer science and art and to present pupils with possibilities of becoming creators of digital media rather than pure consumers. To reach our practical goal a group of researchers and artists designed and implemented a workshop program for children based on the open source software tool Scratch and the artistic idea of ReMida centers based on recycling and reusing waste materials. The workshops have been documented by notes and pictures which serve as data for workshop evaluation.
\end{abstract}

Keywords: Children, art and technology, Scratch, open source software, ReMida, recycle, workshop, new media art, interactive art, creativity, child-computer interaction.

\section{Introduction}

Computer literacy has been proposed to be of equal importance in today's society as literacy that more traditionally refers to people's communicative competencies through skills of writing and reading [6]. The term refers to the ability to understand and to make oneself understood through computational materials. To be fluent in the digital world includes designing and creating and thus goes beyond activities as browsing and interacting [11]. Even if computers have entered schools and are pervasive in the life of young people today, few learn to program [11].

Our argument is that computer literacy can be learnt in a context in which technology is combined with art. To implement this idea, we designed and ran a workshop program with the following goals: 1) strengthen pupils' interest in computer science, 2) strengthen pupils' interest in arts and 3) present creative alternatives of digital media use as opposed to pure consumption of video games.

In this paper we present experiences from designing and conducting a workshop program where children collaboratively engaged in creating interactive artworks that react to events in the physical world. Children used the Open Source Software (OSS) Scratch, home made sensors, and recycled materials. This paper is structured as 
follows. Section 2 presents relevant research. Then Section 3 describes planning and implementation issues of the workshops. Next, Section 4 evaluates the workshop goals based on empirical data and presents implications for future workshops. Section 5 concludes with a further discussion and ongoing research work.

\section{Background}

The graphic object-oriented programming language Scratch has inspired a number of researchers and educators worldwide. Scratch, developed by the Lifelong Kindergarten Group at MIT Media Lab, makes it easy for beginners to get started with creating interactive stories, animations, games, music, and art and share them on the web [11]. Programming in Scratch is done by dragging command blocks from a palette into the scripting pane and assembling them in stacks [7].

Conducting workshops is a common approach in order to introduce groups of children to first programming languages. A lot of research deals with children as users of programming tools, e.g. [10] [3] [13]. Burke and Kafai [4] emphasize the double benefit of supporting both the learning of programming and the learning of writing by situating digital storytelling within the context of programming.

Another study reports on Scratch programming experiences of urban youth in an after-school center and includes an analysis of their use of programming commands and concepts over an 18-month period [7]. The findings related to the experiences of the youth show that they associated Scratch with activities that support creative and personal expression. Peppler and Kafai explored various media arts concepts in workshops conducted at after-school centers in their efforts to bring media arts into design work [10].

\section{The Computer + Art $=$ Creativity Workshops}

Our research concerns exploring the strategy of combining programming activities, recycled materials, and the physical environment to create new media art in workshop settings. The workshop goals, as declared in the application that enabled funding to the project, are: Through encouraging creative expressions in the intersection between art and technology, the goal of the workshop program is to: 1) strengthen pupils' interest in computer science, 2) strengthen pupils' interest in arts and 3) present creative alternatives of digital media use as opposed to pure consumption of video games.

\subsection{Context}

The concrete focus of our workshops is the OSS Scratch and the Reggio Emilia education philosophy of reuse and creativity. Each workshop lasted two days. In the first workshop the participants were 15 pupils from School A. In the second workshop the participants were 14 pupils from school B. All children were 12 years old. The pupils were divided into three groups. They worked in these groups during the whole workshop. Each group had two computers. 
ReMida centers work according to Reggio Emilia education principles [1]. This means that the initiative for creative actions should spring from the child itself. ReMida centers are magic places with a lot of appealing objects where children start to work without being activated by adults. The adults act as assistants.

The workshop theme was 'A Fairytale for the Future'. Fig. 1 depicts the context of the workshop and zooms on two scenes in which the pupils are using Scratch.

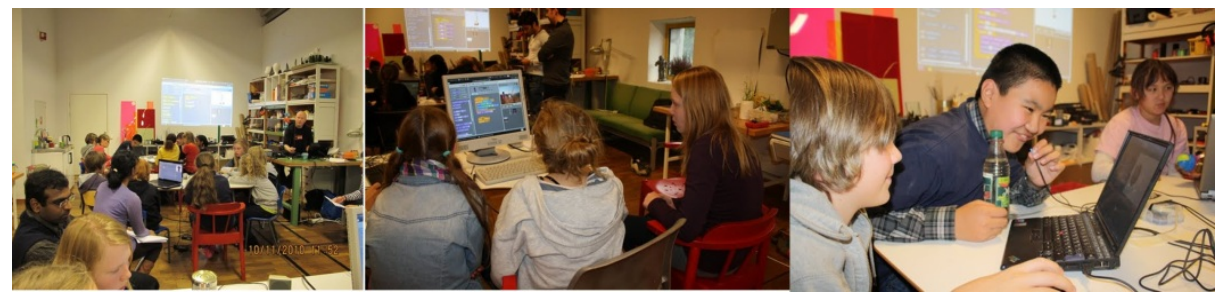

Fig. 1. Left: An overview of the room with the three groups in a tutorial setting; Center: Three girls are using Scratch; Right: Two boys are using Scratch

After studying the available documentation provided at the Scratch website, we designed a plan consisting of nine main steps to be executed in two days.

1. Short demonstration of a Scratch project

2. Make physical characters, take pictures and edit cutout images. Each group was given the task to create two 3-dimensional characters of recycled materials and import digital images of these into Scratch (Fig. 2).

3. Scratch tutorial first part: sprite animation, change costume, movement, sound and graphic effect

4. Make a storyboard and start making scenes in Scratch. A fairytale should be programmed in Scratch and progress as a result of some kind of interactivity between the audience and the artwork triggered by sensors.

5. Scratch tutorial second part: change scenes, synchronization (broadcast and whenreceive), check the value of a variable, actions from sensors.

6. Finish programming. The connections from Scratch to the physical world by means of light, sound, and touch sensors should be implemented by home made sensors connected to the PC by Arduino boards.

7. Decorate a room for exhibition and install artworks (see Fig. 5 center and right).

8. Presentations (See Table 2 and Fig. 5 left).

9. Closing discussion (see Table 3).

Six interactive artworks were made during the workshops. The Scratch projects can be found at the Scratch Web site published under the name artentnu2009. The scripts have been modified so that the projects run without the originally used Arduino boards and connected sensors.

\subsection{Research Method}

Organizers were two artists, one $\mathrm{PhD}$ student, four master students, one senior researcher, and one project manager. One of the artists was the responsible for 

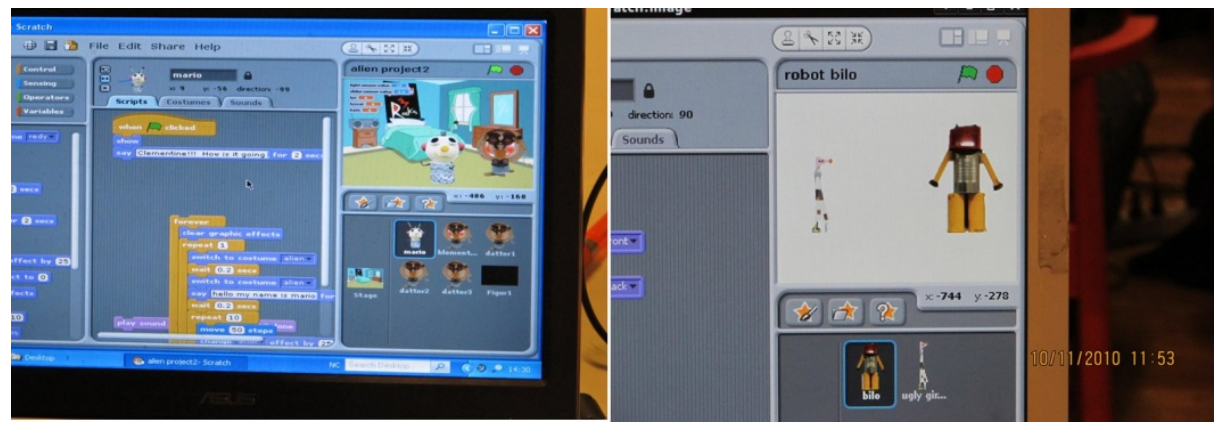

Fig. 2. Giving life to characters in Scratch

ReMida and the other (hereby called the programming artist) has a background as a technology artist. For each group there was an assistant and an observer. The assistants task was to offer help if the pupils had questions and to motivate them during the process.

How to evaluate one person interest in science in general and in computer science in particular is an issue that has been addressed for example in [3] by asking people directly about their interest in programming (respectively art) before and after a science in society event. In our project, there was an initial agreement between the project manager, the two artists, and the senior researcher that this kind of questionnaire based research should not be carried out during the workshop time to avoid stealing time from the creative activities. We agreed that we would take pictures, videos, and notes during the workshops and that the interviews with the pupils should be run after workshop completion.

Data was collected from participant observation. Participant observers take part in proceedings while observing and recording activities and interactions [8]. We took 360 pictures, 197 short video clips, and field notes (all available at [2]). Moreover the produced Scratch projects were saved at the MIT Scratch web site. Concerning permission, we asked the school teachers about the possibilities of taking pictures and publishing them. The teachers replied that all pupils had given permission to the school to allow use and publication of pictures.

Field notes, pictures and video material analysis have been systematically analyzed in order to address the research goals. In the process of selecting pictures we have kept the most relevant pictures and deleted all the pictures we took of the objects to be animated and all the pictures about irrelevant interaction between pupils and master students. We selected 190 pictures which have been classified according to tags like 'Scratch', 'Physical', 'Artist Programmer', 'joy', 'boredom', 'concentration'. All the field notes (recorded by six people) have been collected and transferred into digital form in an observation report. Relevant video material has been selected and transcribed ad verbatim.

\section{Evaluation}

In this section we evaluate the three goals by observing and reflecting about the collected data. 


\subsection{Goal 1 - Strengthen Pupils Interest in Computer Science}

The majority of the pupils had no previous experience with Scratch or similar programming environments. With its simple graphical interface, Scratch proved to be suitable for supporting an exploratory approach from the beginning. The pupils intuitively engaged in testing out the effects of snapping different programming blocks together. During the tutorials some of the basic programming concepts were demonstrated and explained, such as sequence, looping, conditional statements, variables, synchronization, real-time interaction and random numbers. Our notes include several observations of discussions that indicate that pupils developed vocabulary with Scratch terms. This understanding can imply an interest in programming. When the pupils were left to work on Scratch they seemed to enjoy playing with the sound blocks and recording their voices apart from being able to make the sprites move and change costumes. The conversation fragment in Table 1 exemplifies how two pupils used programming terms while working in Scratch.

Table 1. Conversation Fragment, first workshop, day 2, three girls, S, J and V are programming

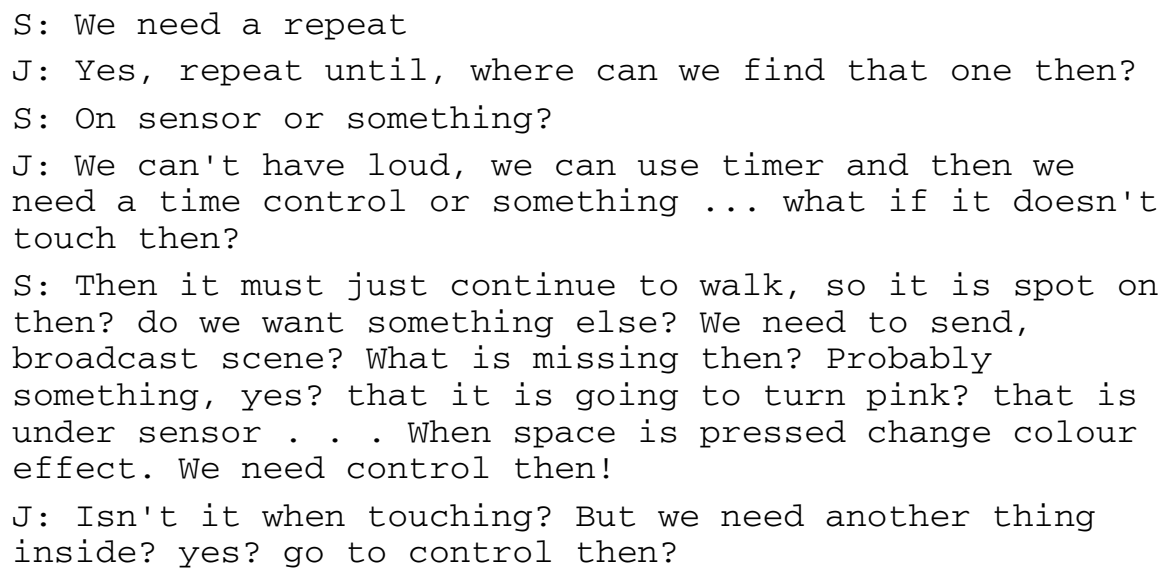

The girls, S and J, use the terms that they see in the Scratch command palette. This conversation demonstrates a learning activity even though it is not possible to evaluate the specific understanding they have of the terms they are using. Other conversation fragments indicate more understanding as the pupils reflect about why a specific behavior is occurring. In the wake of one such reflective discussion, one pupil enthusiastically uttered: 'I am going to download this program at home!'.

Other observations indicate that a number of pupils found it difficult to use Scratch. The second Scratch tutorial introduced concepts that were more challenging to grasp, for instance actions from sensors. In order to clarify how sensors trigger interactive actions, the pupils could have been more included in the process of making the sensors and connecting these to the Arduino board and the computer. One group however, explained well how the audience could interact with their artwork. The fragment in Table 2 illustrates understanding and interest. 
Table 2. Presentations, boy (B) and girl (G) explain their artwork, first workshop, day 2

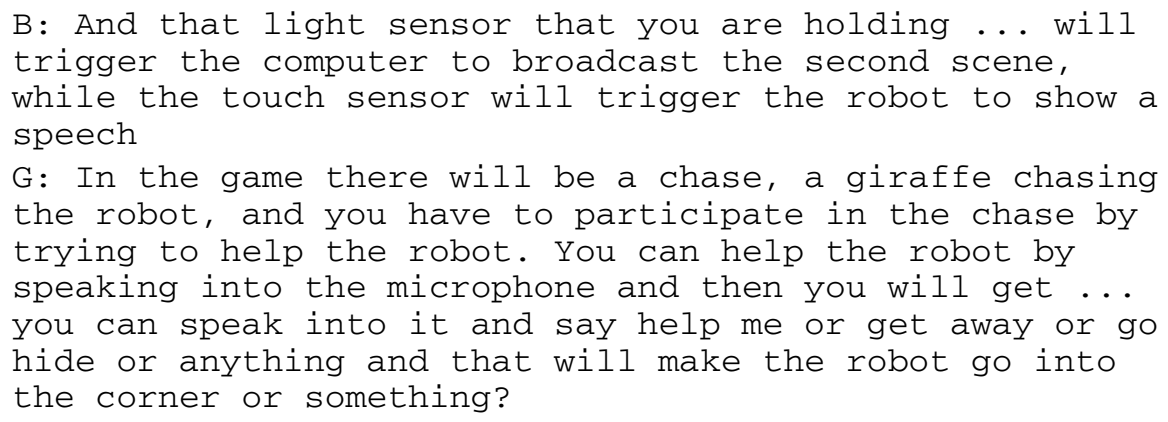

Our transcriptions of conversations between children show that some children are explaining programming concepts in their own words and this demonstrates understanding.

\subsection{Goal 2 Strengthen Pupils Interest in Arts}

ReMida's rich collection of recycled things initiated an immediate creative process within all the groups. The pupils started their projects by making the physical characters. They were experimenting with materials in various shapes, colors and sizes. While the characters were created, the pupils discussed their personality traits and behavior. As the characters gradually got an identity, the story of the fairytale started to develop. This enabled the pupils to get personally connected to their work from the beginning.

Fig. 3 and Fig. 4 respectively show pictures from the activities of making the characters and pictures. We recommend taking pictures of the physical characters different movements as this can be more simple and effective in the process of producing animation compared to using the graphical editor. Furthermore drawing a storyboard proved to be a useful activity for achieving a common understanding of the plot of the fairytale.

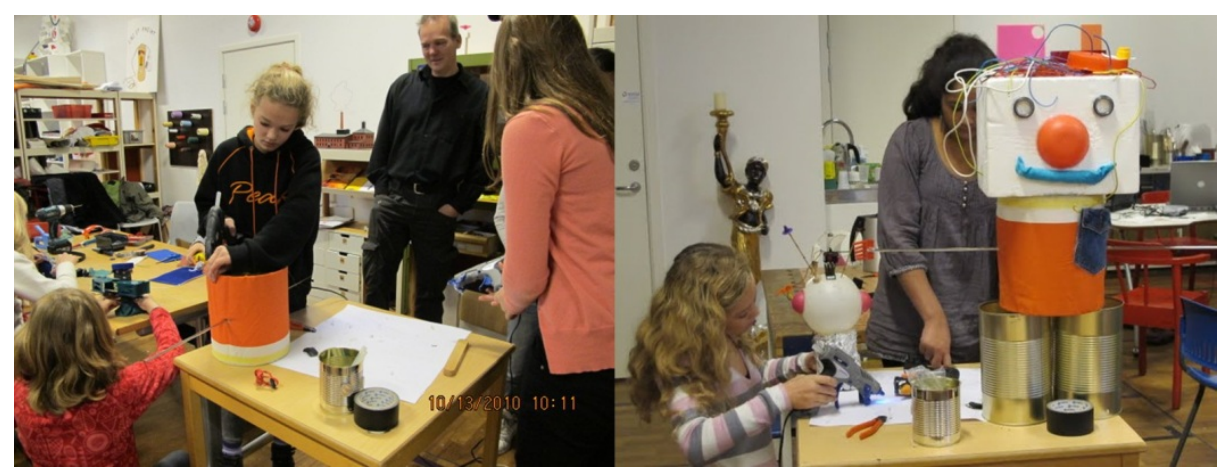

Fig. 3. Pupils work with their physical artifacts 


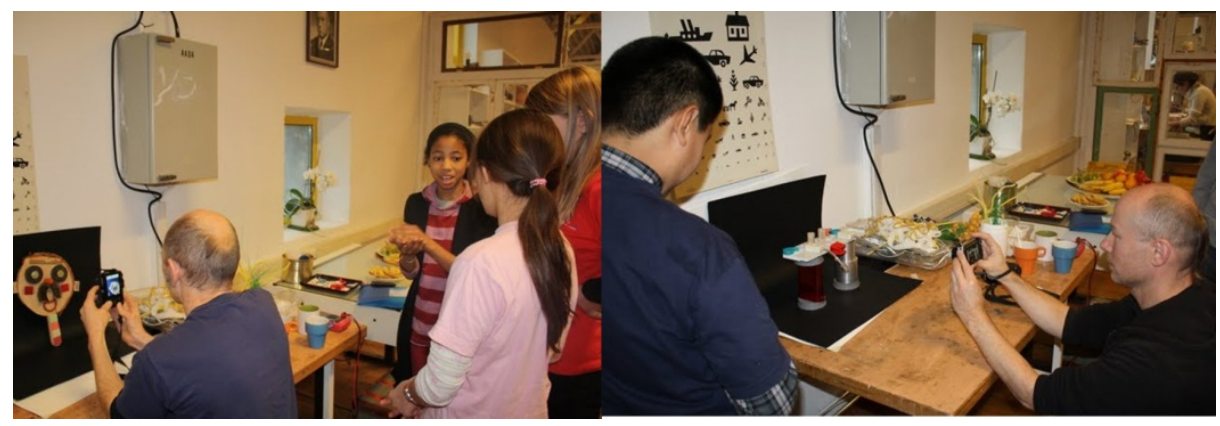

Fig. 4. Artists take pictures of physical characters

Table 3. Closing discussion, second workshop, day 2, PA is programming artist, B is boy, $\mathrm{G}$ is girl and $\mathrm{SC}$ is several children agreeing

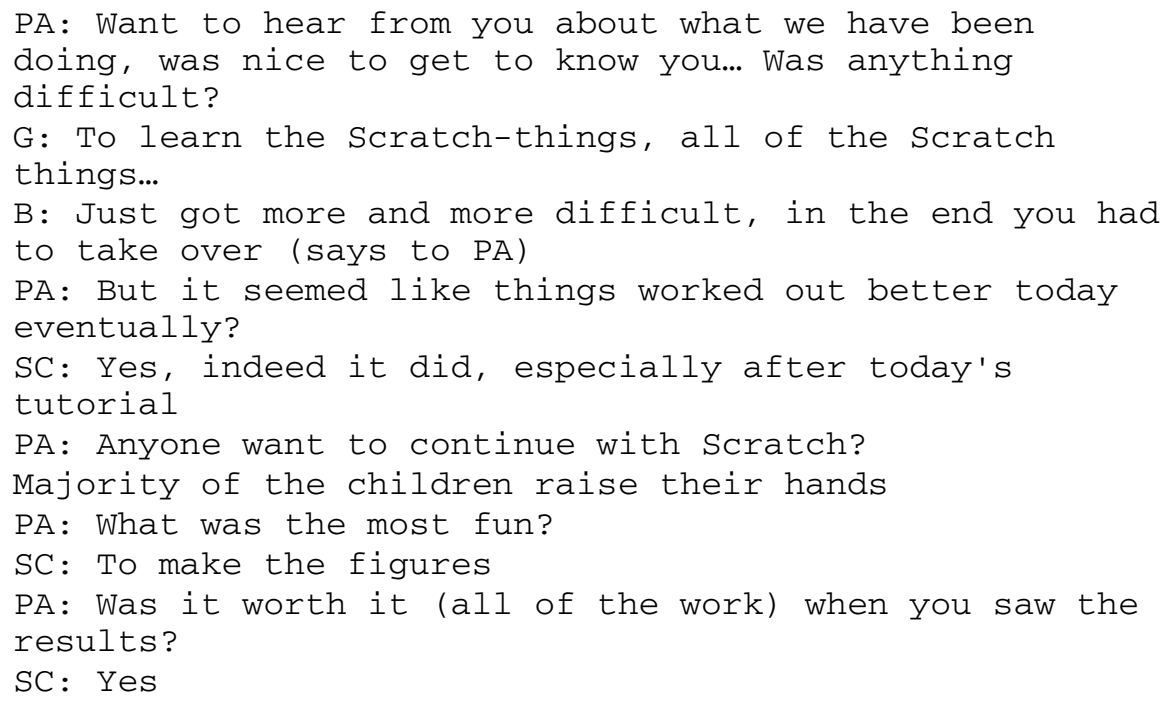

The pupils needed to make a set of artistic choices in Scratch such as deciding which backgrounds, movements, sounds and music to use. Furthermore they had to choose by which means the audience should be able to interact with their artwork. Light, sound and touch sensors were available in order to make the connections from Scratch to the physical world. One of the groups created a fairytale about the alien Mr. Mario and Clementine who fall in love. Each time the light was turned on a new baby was born. During the presentation there was great curiosity to find out whether the next baby would be a girl or a boy. This element of surprise resulted in many babies, but also much laughter and proud faces. In the sea monster project the pupils invited the audience to step on a touch sensor. This action first activated the fairytale to start and then the sea monster to come out of the water. 


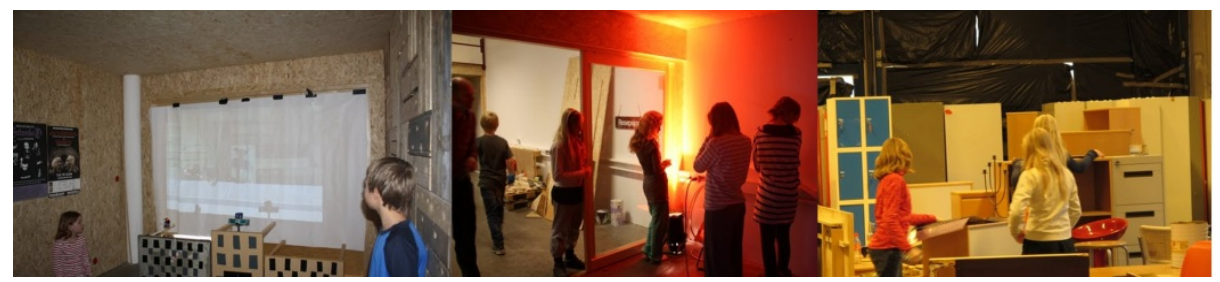

Fig. 5. Left: Presentations; Center and Right: Preparing for exhibitions

The pupils decorated a room for exhibition (Fig. 5). These rooms became important parts of the artworks. The pupils could use all of the ReMida material including a large collection of second hand furniture, smaller interior objects, textiles and different lighting possibilities. Use of these materials enabled the pupils to create the atmosphere they had envisioned for their artwork. A majority of the pupils seemed to really enjoy using the physical space in this manner. One girl excitedly uttered: 'I just love decorating houses. I love decorating everything!' And another girl replied: 'Oh oh oh this is so cool!'.

One of the groups worked especially devotedly to create a specific atmosphere. This group regarded the whole space of the exhibition room as their artwork and created an underwater-like atmosphere that would aesthetically fit the Scratch project. One of their physical characters, the monster boat, was for instance given an extra dimension by attaching its adjustable mouth to a rotating fan to get a continuous open-close movement.

Finally, the pupils were able to share their works and experiences with each other during the presentations (See Fig. 5 left and Table 2). Before allowing the audience to interact with the artworks, the pupils explained what the fairytales were about. Some pupils also explained how they had used Scratch and which sensors they had implemented. As activities, the presentations were successful in order to celebrate the feeling of accomplishment. Furthermore it was valuable for getting information about the pupils overall understanding and reflections from the process.

The pupils seemed to collaborate easier when working with physical materials than when programming. In the closing discussion (see Table 3) many children agreed that the most fun part was to make the figures. An important aspect of the creativity process is to offer pupils a broad variety of artistic elements [5]. Combining Scratch, recycled materials, sensors and physical space is a means to break from conventional expectations by encouraging interest in both art and technology at the same time.

\subsection{Goal 3 Present Creative Alternatives of Digital Media Use}

What was the overall response of the pupils? The workshops in general successfully engaged the pupils as new media artists. In [11] Scratch technology is evaluated to be a driver for digital creativity by giving a message about the positive and creative sides of exploiting such technology in children settings. Our analysis of the pictures shows a situation that is more nuanced than the situations described in [11]. Fig. 6 shows situations in which the boys and girls are happy or playful, tired or bored, and concentrated. Table 4 gives a conversation fragment that can be read together with the left picture in Fig. 6. The fragment and the left picture give evidence to the fact that creative production of new media experiences can be a source of entertainment. 
Table 4. Conversation Fragment, first workshop, day 1, PA is programming artist and A is assistant, two boys, $\mathrm{R}$ and $\mathrm{B}$, are experimenting with controlling the graphic effects by speaking into the microphone
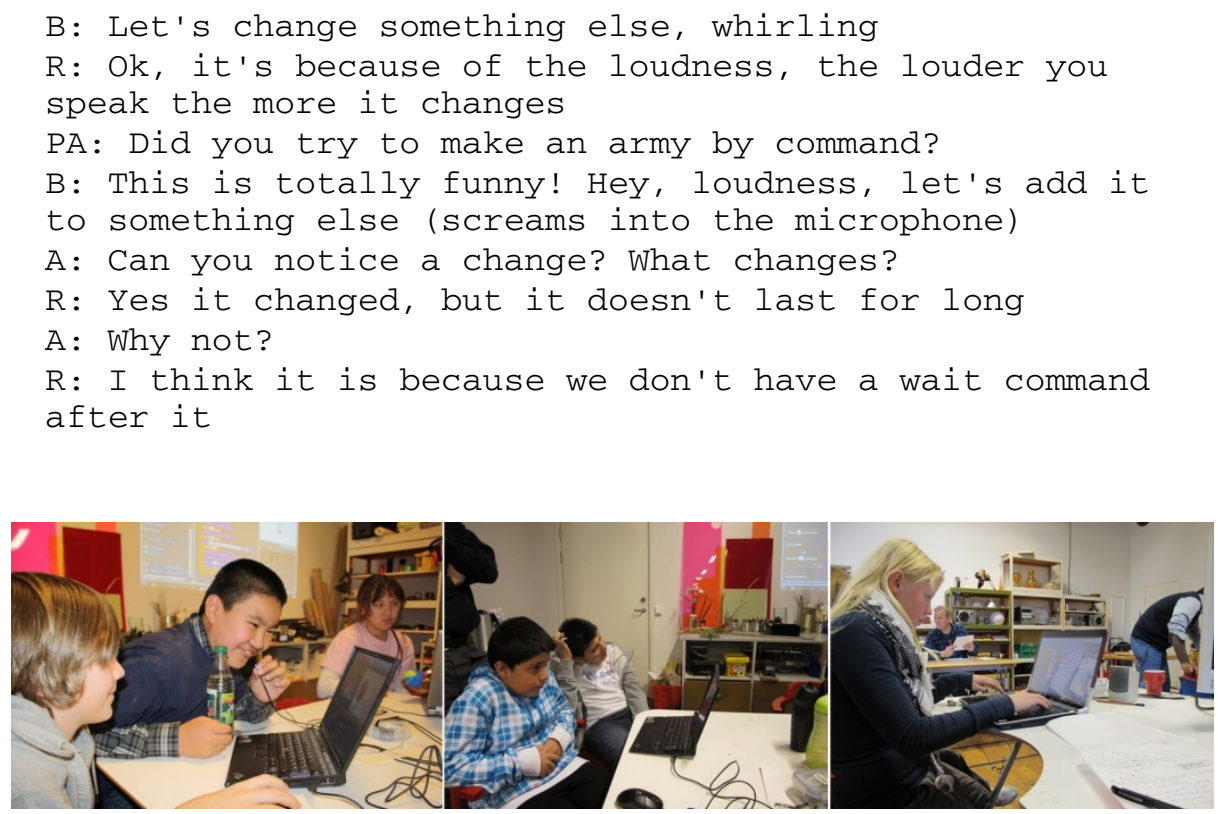

Fig. 6. Happy, bored, and concentrated while engaging in art and technology activities

All tagged pictures are available at [2]. It is possible to count the number of pictures that convey a notion of happiness, concentration and tiredness. However, this information would not be objective as photographers are trained to take pictures of joyful situations so there could be an overrepresentation of these positive feelings. A deep observation of a few pictures and subsequent reflection can bring important insight in the relationship that the individual develop to the making and fruition of the objects of art and technology.

There were noticeable differences between the pupils' ability and motivation to understand concepts in programming activities and motivation. In the first workshop the activities were carried out according to the initial plan. For this group of children this set up seemed to work well. In the second workshop however, we decided to alter the order of two planned activities as it became apparent that several pupils started to lose their motivation and experienced problems during programming the final scenes in Scratch. By starting the activity of decorating the rooms they could still be engaged with the project while being able to use their creative expressions in a different manner. This was a strategy that enabled the children to regain motivation for completing the projects in Scratch. We suggest that workshop organizers can account for different groups of children and different individual interests by designing a workshop activity plan that supports a flexible working style. By this we mean that if the children lose concentration during one activity they should be able to continue 
with a different activity that is still related to the project. Pupils' different interests can be taken into account by offering a variety of tools and activities. Scratch, physical artifacts and sensors make up a set of tools that fulfill different interests when exploring art and technology.

\section{Discussion and Conclusions}

In this paper we presented experiences from designing and conducting a workshop program where children collaboratively engaged in creating interactive artworks by using Scratch, sensors, and recycled materials. We explored the potential of this physical/digital combination as a means to increase pupils' interest in art and technology and to introduce them to become creators of new media artistic expressions.

We can reflect on the digital versus physical dimension by referring to the work in [3] whose goal is to empower collaboration, learning, and design by using digital technology and at the same time taking advantage of human abilities to grasp and manipulate physical objects and materials.

Our findings indicate that combining physical artifacts, programming languages and sensors is a promising approach to increase interest in both art and technology, in a workshop setting. When creating digital art the following three conditions should be strived for: 1) active engagement in the learning process; 2) personal connection and 3) creating projects that are of value to a larger community [9]. The philosophy behind Scratch is a constructionist theory of learning, thus that people learn best when they are active participants in design activities [10]. The ReMida philosophy [1] is similar. Our workshop program did encourage active engagement in learning process. Furthermore there were several levels of expression. First the personal level; second collaboration in groups of two; third cooperation in the group of five: last presentations for the whole class. Findings from our study give the following implications for future workshop programs:

- Choose technology that is low cost and possibly open source in order to encourage children to be able to continue with the activities at home or in school settings. This is motivated by the discussion in Section 4.3.

- Programming concepts should be carefully and pedagogically explained. This guideline may sound obvious. We reflected and discussed on whether the programming concept synchronization (broadcast and when-receive) was too advanced for the available workshop time and also the workshop goals. Interobject communication (communication and synchronization) is one of the most complex ideas in Scratch [7]. Tutorial presentations are not always effective and concepts need to be repeated to individuals and small groups when they struggle with understanding them. We refer concretely to the conversation fragment in Table 4 in which the programming artist and the assistant ask questions to the pupils to make them reflect about what they are doing and learning.

- The process of making own characters (as opposed to only using the ones offered by Scratch) contributes to a more personal connection. This is supported by the evaluation in Section 4.2. 
- Taking pictures of the physical characters different movements can introduce an extra perspective when producing animation compared to using the graphical editor.

- Drawing a storyboard is useful to achieve a common understanding of the story's plot.

- Organizers should design activities that support a flexible working style to account for different groups of children.

- Children should collaborate in subgroups of two when using Scratch. This is supported by the evaluation in section 4.1. Concretely, the fragments in the tables report conversations between couples of children. Here, we refer to the study reported in [12]. An interesting finding of this study is that the highest levels of interest were displayed in situations where the children were working as a team on a goal that they had jointly adopted. This is consistent with the findings of previous studies which suggest that cooperative goal structuring can facilitate learning by providing a context for peer tutoring and by adding social motives such as social responsibility and social approval [12]. In addition, these studies indicated that the optimum learning situation is not one- to-one, but two children to one computer so that they have the opportunity to stimulate each others thinking.

- Letting the children present their projects for each other is an important part of the learning process.

There is an ongoing research work that aims at a further evaluation of the workshops by interviews conducted three months after the workshops. Preliminary results show that the pupils have good memories of the workshop activities and are positive to this kind of activities.

Acknowledgments. Thanks to P. Bøyesen, A. Eriksen, J. Krankenhagen, R. Proto, J. Danado, I. Leite de Oliveira, and S. Nasambu Wasike.

\section{References}

1. The Hundred Languages of Children: The Reggio Emilia Approach Advanced Re-ections, 2nd edn. Ablex Publishing, New Jersey (1998)

2. ArTe-team: Arte's blog and content management system (March 2011),

http: / /www. artentnu.com

3. Buechley, L., Eisenberg, M., Catchen, J., Crockett, A.: The lilypad arduino: us- ing computational textiles to investigate engagement, aesthetics, and diversity in computer science education. In: Proceeding of the Twenty-Sixth Annual SIGCHI Conference on Human Factors in Computing Systems, CHI 2008, pp. 423-432 (2008)

4. Burke, Q., Kafai, Y.B.: Programming \& storytelling: opportunities for learning about coding \& composition. In: IDC 2010, pp. 348-351 (2010)

5. Edmonds, E., Candy, L.: Creativity, art practice, and knowledge. Commun. ACM 45(10), 91-95 (2002)

6. Fernaeus, Y., Tholander, J.: Three challenges when designing for childrens everyday digital literacy. Nordic Journal of Digital Literacy 2, 130-139 (2008)

7. Maloney, J.H., Peppler, K., Kafai, Y., Resnick, M., Rusk, N.: Programming by choice: urban youth learning programming with scratch. In: Proceedings of the 39th SIGCSE Technical Symposium on Computer Science Education, SIGCSE 2008, pp. 367-371 (2008) 
8. Oates, B.J.: New Frontiers for Information Systems Research: Computer Art as an Information System. European Journal of Information Systems 15 (2006)

9. Peppler, K.A.: Media arts: Arts education for a digital age. Teachers College Record 112(8), 2118-2153 (2010)

10. Peppler, K.A., Kafai, Y.B.: Youth as media art designers: Workshops for creative coding. Tech. rep., Chicago, IL (2008)

11. Resnick, M., Maloney, J., Monroy-Hernandez, A., Rusk, N., Eastmond, E., Bren-nan, K., Millner, A., Rosenbaum, E., Silver, J., Silverman, B., Kafai, Y.: Scratch: programming for all. Commun. ACM 52, 60-67 (2009)

12. Subhi, T.: The impact of LOGO on gifted children's achievement and creativity. Journal of Computer Assisted Learning 2(15), 98-108 (1999)

13. Tangney, B., Oldham, E., Conneely, C., Barrett, S., Lawlor, J.: Pedagogy and processes for a computer programming outreach workshop - The bridge to college model. IEEE Transactions on Education 1(53), 53-60 (2010) 